\title{
Hermite sieve as a wavelet-like array for I D and 2D signal decomposition
}

Y.V. Venkatesh

K. Ramani

R. Nandini

Indexing terms Wavelet transform. Multiresolution, Signal decomposition, Generalised Hermite pol ynomials, Scale space, Fourier series, Windowed Fourier transform, Zero crossings

Abstract: A new class of an array of wavelet-like functions, derived from generalised Hermite polynomials and controlled by a scale parameter, has been used to create a multilayered representation for one- and two-dimensional signals. This representation, which is explicitly in terms of an array of coefficients, reminiscent of Fourier series, is stable. Among its other properties, $(a)$ the shape of the resolution cell in the 'phase-space' is variable even at a specified scale, depending on the nature of the signal under consideration; and $(b)$ zero crossings at the various scales can be extracted directly from the coefficients. The new representation is illustrated by examples. However, there do remain some basic problems with respect to the new representation.

\section{Introduction}

The motivation for multiscale, multichannel analysis ha come from the results of psychophysical experiments on biological vision [1-3]. A plausible hypothesis for this type of decomposition is that the structures or details in the physical world constituting the input to the sensory system have many different sizes. However, in natura vision (unlike computer vision systems) information extraction and recognition of object details seem to be independent of image scale. Motivated by this discovery due, among others, to Hubel and Wiesel [4], some recent investigations in the area of computer vision have deal with the problem of representation of an image in severa frequency channels. Scale space representations and analysis [5] are based on the idea that different characteristics of a signal reveal themselves at different levels of resolution or, equivalently, in several frequency channels. When the signal includes important structures that belong to different scales, it is useful to split the signal information into a set of components of varying size. In the course of such a decomposition, it is imperative, fo stability, that a small perturbation of the representatio reflects a small modification of the original signal. At the same time, it is also desirable to localise spectral information in the signal. This appears to be the principal motivation for signal decompositions based on either windows or frequency channels, A desirable characteristic of any signal representation scheme is that it should enable us to extract signal properties.

An image can be treated as a stacking of onedimensional scan lines, and the mathematical preliminaries presented for one-dimensional signal analysis can, in principle, be extended to image analysis. However, the framework for two-dimensional signal analysis is definitely more complicated than that of one-dimensional signal analysis. For instance, zero-crossing information is contained in a set of discrete joints for one-dimensional signals, as against contours (which contain infinite points) for two-dimensional signals. See, for instance, Curtis, Oppenheim and Lim [6], in which these authors treat the two-dimensional signal synthesis problem as characteristically different from that of the one-dimensional signal synthesis. Further, the bandpass characteristics of twodimensional signals cannot be analysed in terms of their projections on frequency axes. An image filtered through a (bandpass) bar-shaped mask is band-pass on each scanline perpendicular to the mask's orientation; whereas, an image filtered through a (bandpass) circularly-symmetric mask is band-limited but not band-pass along any scanline. This follows from the fact that the Fourier transform along (for instance) the $\mathrm{x}$-axis of an image filtered through a bandpass 'ring' is essentially the projection of the two-dimensional Fourier transform on $\omega_{x}$, and is therefore not bandpass. Further, the concept of neighbourhood is based on regions in two dimensions as opposed to intervals in one dimension. Orientation of features has no counterpart in one dimension, but is an important aspect of two-dimensional signals. Above all, the human vision System serves as a paradigm for developing appropriate models for representation and analysis $[7,8]$ 


\subsection{Representation of signals}

Given a sequence of increasing resolutions $\left(r_{j}\right)_{j \in Z}$, the details of a signal at the resolution $r_{j}$ are defined as the difference of information between its approximation a the resolution $r_{j}$ and its approximation at the lower resolution $r_{j-1}$.

A structure for implementing this scheme is called the pyramid $[9,10]$, which is a sequence of signals in which each is a filtered version of its predecessor. Each signal in the sequence is represented by an array which is half the size of its predecessor. The filtered signal is represented at reduced resolution and sample densities. Assuming a function $f(\cdot)$, one can define the pyramidal structure as a collection of subsampled signals connected by a mapping transformation. Local operators of many scales but identical shape serve as the basis functions. The operations include low and bandpass filters and window functions. Implementation of such an approach includes blur (or reduce), expand (to make two levels of the pyramid compatible in size), and difference (to subtract). However, a disadvantage of such a representation is that the elements of the signal sequence are correlated.

An approach to the extraction of localised spectra information is the use of Fourier analysis in a window of the signal. This results in a representation which is intermediate between a spatial and a frequency description. Use of a Gaussian window minimises the uncertainty associated with the spatial-spectral resolution, as exemplified by the results of Marr [7], which for 2D signals (images) involves the filtering of the original signal with the Laplacian of a Gaussian for various values of the variance parameter. In this case, the multiscale representation is a multichannel representation in the frequency domain where a channel corresponds to some specific passband. However, the size of the resolution cell in such a representation is fixed, and, therefore, the finer details in a signal when interspersed with coarse information, cannot be separated out satisfactorily.

\section{Wavelet transforms}

To overcome the limitations of the windowed Fourier transform, a combined spatial-spectral representation $\grave{a}$ la Gabor [11] or the so-called wavelet transform has been proposed. The Gabor scheme uses a modulated version of the Gaussian, but, unfortunately, the Gabor functions do not constitute an orthogonal basis. More importantly, it is also known that they are not easily amenable to an orthogonalisation procedure for extracting the coefficients of the signal in the Gabor space [12].

On the contrary, the wavelet transform is computed by expanding the signal into a family of functions which are the dilations and translations of a unique function, $\phi(x)$, called a wavelet. Grossman and Morlet [13] decompose a function in $L^{2}(R)$ using the family of functions:

$[\sqrt{ }(s) \phi(s x-b)]_{(s, b)} \in R^{+} \times R$

A wavelet transform is then interpreted as a decomposition of the given signal into a set of frequency channels having the same bandwidth on a logarithmic scale.

Consider an one-dimensional signal in $L^{2}$. Let $\phi$ denote a function with sufficient decay, say $\|\phi(x)\| \leqslant$ $c /\left(1+x^{2}\right)$, with

$$
\int_{-\infty}^{\infty} \phi d x=0
$$

In what follows, $\phi_{s}(x)$ denotes the dilation of $\phi(x)$ by a factor ' $s$ ' and $\phi_{s}^{a}(x)$ denotes the translation of $\phi_{s}(x)$ by a factor ' $a$ ':

$$
\phi_{s}(x)=\frac{1}{s} \phi(x / s) \quad \phi_{s}^{a}=\frac{1}{s} \phi\left(\frac{x-a}{s}\right)
$$

Such a function is called a wavelet. The wavelet transform of a signal is given by correlating $f$ with $\phi_{s}^{a}$.

$$
W f(s, a)=\int_{-\infty}^{\infty} f(x) \phi_{s}^{a}(x) d x
$$

The choice of $\phi$ determines the compactness of the representation, and the inversion is achieved by an appropriate inverse integral. In practice, for computational ease most commonly, $s$ and $a$ are restricted to some discrete subset: $s=\mathbf{2}$ and $\mathbf{a}=2^{-n}$ with $m, n \in \mathscr{Z}$, generating a set of dyadic wavelets. An example of an orthonormal basis for discrete wavelets for $L^{2}(\mathscr{R})$ is the Haar basis.

\subsection{Choice of the wavelet function}

The choice of the wavelet function $\phi$, has been the subject of many investigations. It is found that creating an orthonormal basis of $L^{2}(R)$ is quite involved. Some authors use a function which is similar to the Laplacian of the Gaussian (LoG), and others have tried to generate wavelets by recursive procedures. Common to all these attempts is the difficulty in generating orthogonal functions for a unique representation of the given signal. For instance, Mallat [14c] speaks of an orthonormal basis of $L^{2}(R)$ generated by the family of functions,

$$
\left[\phi_{2}^{j}\left(x-2^{\prime} n\right)\right]_{(n, j, \in Z \times Z}
$$

However, in practice, the standard procedure adopted, for computation, is a decomposition of the signal using the so-called 'quadrature mirror' filters [14]. This skirts around the wavelet representation by avoiding the computation of coefficients. In this approach, the signal is represented using a finite set of resolutions in powers of 2. The basic idea is to separate the higher and lower halves of the spectrum of a signal by using second order bandpass and low-pass filters. Then the signal is subsampled corresponding to the lower half of the spectrum. This procedure is applied iteratively. This is equivalent to dividing the spectrum into successive bands, and extracting the details corresponding to these bands. Suppose, for instance, that the original signal is at resolution 2 . The result of the first band pass filtering will give us the difference of information between resolution $2^{j}$ and $2^{j-}$. The next band pass filtering will give us information between $2^{j-1}$ and $2^{j^{-2}}$, and so on

Zak [16] discusses a quantum mechanical representation (called the kq-representation), based on the quasimomentum $\mathrm{k}$, and the quasicoordinate $q$. The wavelet basis functions of Zak are given by

$$
\psi_{k q}(x)=\sqrt{\left(\frac{2 \pi}{a}\right)} \sum_{n} e^{j k n a} \delta(x-q-n a)
$$

where $\delta(\cdot)$ is the Dirac-delta function. The Zak transform is then defined as

$$
(U f)(k, q)=\sqrt{\left(\frac{a}{2 \pi}\right) \sum_{n} e^{j k n a} f(q-n a)}
$$

where, for any $q$, only a finite number of terms in the sum contribute, in view of the assumption of compactness of the support of $f$. The Zak transform is similar to the Gabor transform except that modulated and shifted 
Dirac-delta functions are used in place of the modulated Gaussian function, and the transform is obtained as an infinite sequence. For a recent reference to this transform, see Daubechies et al. [17]. However, it is not obviou from these references how one can obtain, in practice, a signal representation which really exhibits localisation in both the time/space and frequency domains.

A recent contribution* to the literature is the use of psi-transforms for filtering the signal into low-pass an band-pass components [18]. The set of analysing and synthesising functions is generated as a solution to an optimisation problem: determine a function which is compactly supported in one domain, and 'concentrated' in the other. Examples of this kind of function are the prolate spheroidal wave functions, which are strictly band limited on the frequency interval $[-\boldsymbol{B}, \boldsymbol{B}]$, and have the maximum fraction of their energy in the time/ space interval $[-T / 2, T / 2]$. However, the actual (basic) function generated by such a procedure is very similar to the Laplacian of the Gaussian (LoG) in the time/space domain. Since a solution to the optimisation problem leads to an eigen-function problem, and the solution itself is discontinuous in the frequency domain, a smoothing operating by a Gaussian function is necessary.

The latest paper [19]+ by Newland deals with a harmonic wavelet, which is concentrated locally around the origin, and is orthogonal to its own unit translations and octave dilations. Moreover, its frequency spectrum is confined exactly to an octave band, so that it is compact in the frequency domain (rather than the spatial/time domain). The implications in the resolution space for this type of representation are not clear.

\section{The new vector wavelet transform}

In practice, signals which are spatially finite are not strictly finite in extent in the spectral domain. In order to develop a consistent mathematical theory, we treat signals as having unbounded support in both the spatial and time domains, but, more importantly, we use some measures to indicate the extent of effective signal spread in these domains.

We employ generalised Hermite polynomials to represent the given signal in multiple channels, each channel corresponding to a specific value of the scale parameter $\sigma$ for $I D$ signals, and $\left(\sigma_{1}, \sigma_{2}\right)$, along the $x$ - and y-directions, respectively, for $2 D$ signals. For each channel, the representation, in contrast with the results of the literature, i explicitly a vector or matrix of coefficients, for $1 D$ and $2 D$ signals, respectively. Further, the number of channel is dependent on the amount of residual error permitted in the representation of the signal.

For the sake of simplicity and clarity, we first conside the new scheme of representation of one-dimensional signals, and later indicate its extension to twodimensional signal analysis. The signals under consideration are defined over $(-\infty, \infty)$ in both the spatial and spectral domains. Let $f(x) \in L^{2}(\mathscr{R})$ be a real-valued function of $x \in \mathscr{R}$ with the Fourier transform,

$$
F(j \omega)=\int_{-\infty}^{\infty} f(x) e^{-j \omega x} d x
$$

The two functions, $f(x)$ and $F(j \omega)$, form a Fourier integral pair. The classical uncertainty principle says that they cannot both have compact support or, in other words, be highly concentrated in a finite region of the $x$ and the w-domains [20-22]. A measure of localisation is effective width, whose definition requires the following concepts. The uncertainty inequality can then be obtained by defining the spatial and spectral spreads of the function as follows.

The energy $E$, in a signal $f(x)$, is given by

$$
E=\int_{-\infty}^{\infty}|f(x)|^{2} d x=\frac{1}{4 \pi^{2}} \int_{-\infty}^{\infty}|F(\omega)|^{2} d \omega
$$

The effective widths in the spatial and spectral domains are then given by

$$
\begin{aligned}
& X_{e}=\sqrt{\left[\frac{\int_{-\infty}^{\infty} x^{2}|f(x)|^{2}}{E} d x\right]} \\
& W_{e}=\frac{1}{2 \pi} \int\left[\frac{\int_{-\infty}^{\infty} \omega^{2}|F(\omega)|^{2}}{E} d \omega\right]
\end{aligned}
$$

Using the Schwarz inequality, we obtain the standard 'uncertainty' inequality [22]: $X_{e} W_{e} \geqslant \frac{1}{2}$.

Remark 3.1: Images, which are treated as twodimensional functions, are assumed to be defined over $(-\infty, \infty) \times(-\infty, \infty)$ in both the spatial and spectral domains. Similar 'uncertainty' inequalities in various directions can be derived. However, it is interesting to note that the uncertainty inequalities in two dimensions only come pairwise, along the corresponding axes. Thus we have the space-bandwidth products satisfying the inequalities, $X_{e_{x}} W_{e_{x}} \geqslant \frac{1}{2}$ and $X_{e_{y}} \boldsymbol{W}_{e_{y}} \geqslant \frac{1}{2}$, while $X_{e_{x}} \boldsymbol{W}_{e_{y}}$ can vanish. (Here the use of sub-subscripts is selfexplanatory.)

\subsection{Choice of basis functions}

Consider the generalised one-dimensional Hermite polynomials parametrised by $\mathbf{u}$, and generated as follows:

$$
\begin{aligned}
& H_{n}(z, \sigma)=\left.(-1)^{n} e^{x^{2} / 2} \frac{d^{n}}{d x^{n}}\left(e^{-x^{2}}\right)\right|_{I=z / \sqrt{ }(\sigma)} \\
& \text { for } n=0,1,2,3, \ldots
\end{aligned}
$$

It is known $[23,24]$ that the $H_{n}$ s form a complete basis for the class $C$ of real functions $\psi(x)$, defined on the infinite interval $(-\infty, \infty)$, which are piecewise continuous in every finite subinterval $[-a, a]$ and satisfy the condition

$$
\int_{-\infty}^{\infty}\left(1+x^{2}\right) e^{-x^{2} / \sigma} \psi^{2}(x) d x<\infty
$$

The first few polynomials are:

$$
\begin{aligned}
& H_{0}(x, \sigma)=e^{-x^{2} / 2 \sigma} \quad H_{1}(x, \sigma)=\frac{2 x}{\sqrt{ }(\sigma)} H_{0}(x, \sigma) \\
& H_{2}(x, \sigma)=\left(\frac{4 x^{2}}{\sigma}-2\right) H_{0}(x, \sigma) \\
& H_{3}(x, \sigma)=\left[\frac{8 x^{3}}{\sigma \sqrt{ }(\sigma)}-\frac{12 x}{\sqrt{ }(\sigma)}\right] H_{0}(x, \sigma)
\end{aligned}
$$

which are orthogonal on $-\propto<x<\infty$. Further, they can be shown to satisfy the recurrence relation [24],

$$
x H_{n}(x, \sigma)=\sqrt{ }(\sigma)\left[\frac{1}{2} H_{n+1}(x, \sigma)+n H_{n-1}(x, \sigma)\right]
$$


An important property of these polynomials which facilitates multiscale/multichannel decomposition of signals is that their Fourier transforms are related in a very simple way to the polynomials themselves. Their transforms are given by

$$
\tilde{H}_{n}(j \omega, \sigma)=(-j)^{n} H_{n}(\omega \sigma, \sigma)
$$

Fig. IA shows the first four polynomials for a fixed value of $\sigma$, and their Fourier transform magnitudes are shown in Fig. 1B. Fig. 1C shows $H_{1}(x, \sigma)$ for different values of $\sigma$. Note that the parameter $\sigma$ controls the essential width of the signal in both the spatial and frequency domains; the smaller the value of $\sigma$, smaller the spatial width and greater the spectral width, and vice versa.

In what follows, $\sum$ denotes summation with respect to $n$ ranging, unless otherwise indicated, from 0 to $\infty$. Let the $L_{2}$ norm squares of these polynomials be denoted by $k_{n}^{2}$, for $n=0,1,2, \ldots, \infty$.

A function $f(x) \in C$, is completely specified by the coefficients, $\gamma_{n}$, in the expansion,

$$
f(x) \simeq \sum \gamma_{n} H_{n}(x, \sigma) \quad-\infty<x<\infty
$$

where the coefficients $\gamma_{n}$ are calculated from the relation.

$$
\gamma_{n}=\frac{1}{k_{n}^{2}} \int_{-\infty}^{\infty} f(x) H_{n}(x, \sigma) d x
$$

for $n=0,1,2, \ldots, \infty$. In practice, we use only a finite number, $N$, of terms, and the coefficients $\gamma_{n}$ are obtained from eqn. 2 . The (approximate) signal reconstructed from these coefficients will not match the original at all the individual points. However, theoretically, if infinite term are used in expr. 1, and if the real function $f(x)$ defined on the infinite interval is piecewise smooth in every finite interval $[-a, a]$, and if the integral,

$\int^{\infty}$

is finite, then the series (expr. 1) with coefficients calculated from eqn. 2 converges to $f(x)$ at every continuity point of $f(x)$.

Remark 3.2: Note that the first term in the expansion (eqn. 1) is concentrated (or has its maximum) at $x=0$. The (local) maxima/minima of first and higher order terms are located away from the origin.* It can be shown that the distance from the origin to these locations (distinct from $\mathrm{x}=0$ ) is directly proportional to the order (for fixed scale factor, $\sigma$ ), and to $\sqrt{ }(\sigma)$ (for fixed order) When only a finite number of terms are used at each scale, and the signal is represented at multiple scales, the individual behaviour of the basis functions at higher scales accounts for the convergence of the series expansion (eqn. 1) to $f(x)$ for large frequencies which are concentrated in the spatial/time domain around the origin. On the other hand, large magnitude but low frequency parts of the signal at distant locations can be handled by choosing a large initial value of the scale factor. However, large frequency, large magnitude parts of signal far away from the origin cannot, in practice, be represented satisfactorily by these lower order terms. Therefore, one has to have recourse to shifting the origin to the appropriate point. Note that this is, in effect, windowing the original signal. An alternative strategy is to first embed the given signal in a larger background, locate the maxima of the signal, and then choose the number of coefficients and the value of the initial scale factor appropriately.

Assume that the given signal is expanded in terms of a finite number $(N)$ of the generalised Hermite polynomials,

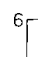

A

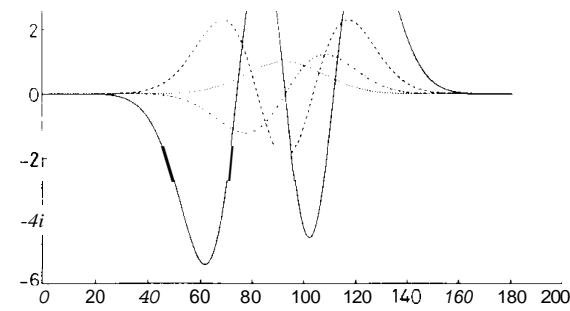

Fig. 1 A Plot of first four Hermite polynomials for fixed a

$H_{0}(x, \sigma)$
$H,(x, \sigma)$

$\longrightarrow H_{3}(x, \sigma)$

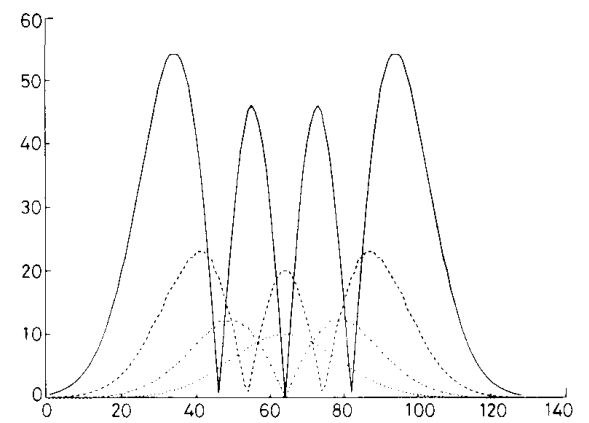

Fig.1B Plot of Fourier transforms (magnitude) of the first four Hermite polynomials for fixed a

$\tilde{H}_{\sigma}(x, \sigma)$

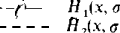

$\begin{array}{ll}- & A_{2}(x, \sigma) \\ - & A_{3}(x, \sigma)\end{array}$

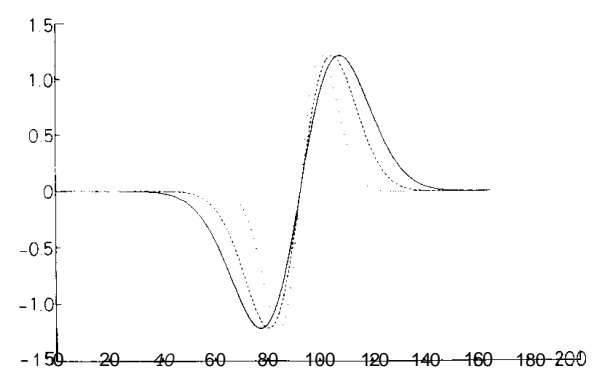

Fig. 1C Plot of second Hermite polynomial $\left[H_{1}(x)\right]$ at three scales: $\sigma_{0}>\sigma_{1}>\sigma_{2}$ 
using the scale parameter $\sigma_{0}$. Then, from expr. 1 we have $f_{\text {approx }}(x)=\sum \gamma_{n} H_{n}\left(x, \sigma_{0}\right)$

$$
\infty<x<\infty \quad n=0,1,2 \cdots N-1 \quad(3)
$$

As indicated above, in view of the fact that we have used only a finite number of terms in the representation (with the coefficients obtained from eqn. 2), the error in the representation at scale $\left(\sigma_{0}\right)$, at any point $x$, is given by

$\operatorname{err}\left(x, \sigma_{0}\right)=f(x)-f_{\text {approx }}(x) \quad x \in \mathscr{A}$

where the error is explicitly shown as dependent on $\sigma_{0}$ This error is orthogonal to $H_{i}\left(x, \sigma_{0}\right)$, for $\mathrm{i}=0,1, \ldots$, $\mathrm{N}-1$. An expansion of err $\left(x, \sigma_{0}\right)$ using $\sigma$ smaller than $\sigma_{0}$ would lead to a representation of remaining components which are at frequencies different from those of $f_{\text {approx }}(x)$. Similarly, the error in the representation at scale $\sigma_{1}$, at any point $\mathbf{x}$, is given by

$\operatorname{err}\left(x, \sigma_{1}\right)=\operatorname{err}\left(x, \sigma_{0}\right)-\operatorname{err}_{\text {approx }}\left(x, \sigma_{1}\right)$

By combining the above equations, it can be shown that

$$
\begin{aligned}
f(x)= & f_{\text {apprax }}(x)+\operatorname{err}_{\text {approx }}\left(x, \sigma_{0}\right) \\
& +\operatorname{err}_{\text {approx }}\left(x, \sigma_{1}\right)+\ldots+\text { residual error }
\end{aligned}
$$

The residual error is the final error which, for all practical purposes cannot be represented because either it is too small or it is beyond the spectral reach of the layered representation. By virtue of the multistage decomposition (Fig. 2), the spectrum of $f_{\text {approx }}(x)$ does not include that of

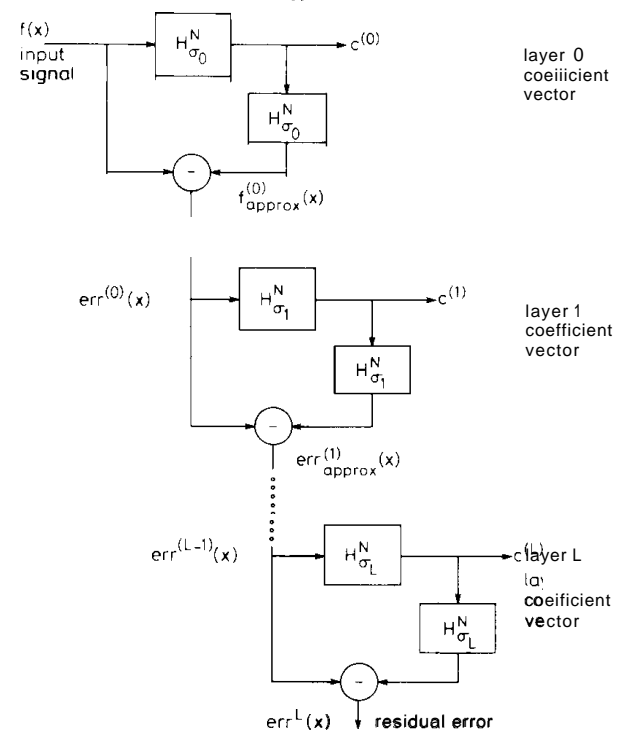

Fig. 2 Block schematic of 10 signal decomposition procedure using vector array of wavelets

$\operatorname{err}_{\text {approx }}\left(x, \sigma_{0}\right)$, which in turn does not contain that of $\operatorname{err}_{a p p r a x}\left(x, \sigma_{1}\right)$, and so on. This is equivalent to applying a sieve of Hermite polynomials at every level. Note that the spectral content retained at each stage is controlled by the scale parameter $\sigma$.

Remark 3.3: When mixing the scale, the Hermite polynomials are no longer orthogonal. The set of Hermite polynomials across multiple scales corresponds to what one calls a frame. The representation proposed results in a decomposition of signals using a redundant set of functions, and is hence robust. However, the problem of how to find a good representation (among the all possible representaticns) using the Hermite polynomials is not resolved in this paper.

Remark 3.4: The generalised one-dimensional Hermite polynomials parametrised by $\sigma$, can be used to generate (by tensor product) the two-dimensional versions, parametrised by $\sigma_{1}$ and $\sigma_{2}$ :

$$
H_{m . n}\left(x, y, \sigma_{1}, \sigma_{2}\right)=P_{m}\left(x, \sigma_{1}\right) P_{n}\left(y, \sigma_{2}\right)
$$

form, $n=0,1,2, \ldots, \infty$.

\section{Properties of the new vector wavelet transform}

We summarise the important properties of the new representation scheme. Mathematical expressions quantifying some of these properties are given here only for 1D signal representation. Their derivation and further explanatory details (including those for $2 \mathrm{D}$ signals or images) are found in Reference $26 c$

Property 1 : If the coefficients are subject to a small perturbation, the resulting signal is also perturbed, and the change in the signal is bounded. Conversely, if the original signal is perturbed by a small amount, then the corresponding change in the coefficients is also bounded. Hence the representation is stable.

Property 2: The shape of the resolution cell depends upon both the spatial spread and the frequency content of the signal. The relations listed below determine the size of the resolution cell in 'phase-space'. The effective spatial spread. $X_{\text {spatial }}$, is defined by

$$
\begin{aligned}
& \qquad X_{\text {spatial }}^{2}=\frac{\int_{-x}^{x} x^{2} f^{2}(x) d x}{E} \\
& \text { where } \\
& \qquad \begin{aligned}
E & =\int_{-x}^{\infty} f^{2}(x) d x \\
& =\sum_{n} \gamma_{n}^{2} \int_{-\infty}^{\infty} H_{n}^{2}(x, \sigma) d x
\end{aligned}
\end{aligned}
$$

By employing the recurrence relations, it can be shown that

$$
X_{s p a t i a l}^{2}=\frac{1}{4} \sigma \frac{\sum_{n}\left[2(n+1) \gamma_{n+1}+\gamma_{n-1}\right]^{2 *} A}{\sum \gamma_{n}^{2} * A}
$$

Similarly, with

$$
\begin{aligned}
& B=\int^{\infty}\left|\tilde{H}_{n}(j \omega, \sigma)\right|^{2} d \omega \\
& B=\frac{1}{\sigma} * A
\end{aligned}
$$

and

$$
k_{n}^{2}=\sqrt{(\sigma) k_{n}^{\prime 2}}
$$

where

$$
k_{n}^{2}=\int_{-\infty}^{x} H_{n}^{2}(x, 1) d x
$$


it can be shown that the effective spectral width $X_{\text {spectral }}$, is given by

$$
X_{\text {spectral }}^{2}=\frac{1}{4 \sigma} \frac{\sum_{n}\left[2(n+1) \gamma_{n+1}-\gamma_{n-1}\right]^{2} * B}{\sum \gamma_{n}^{2} * B}
$$

It can be shown that the effective space-bandwidth product (SBP) is given by

$$
\begin{aligned}
& X_{\text {spatial }} X_{\text {spectral }} \\
& =\frac{1}{4} \int\left\{\frac{\sum_{n}\left[2(n+1) \gamma_{n+1}+\gamma_{n-1}\right]^{2} k_{n}^{\prime 2}}{\sum_{n} \gamma_{n}^{2} k_{n}^{\prime 2}}\right\}
\end{aligned}
$$

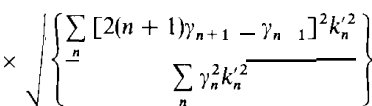

and the space-bandwidth ratio (SBR) by

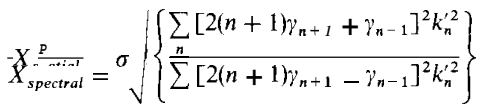

From the last result, we conclude that SBR is directly proportional to $\sigma$, the constant of proportionality bein governed by the ratio of the two quadratic forms which involves the coefficients $\gamma_{n}$, of the expansion. Note that the ratio term inside the square root symbol, though explicitly containing $\sigma$, is actually independent of it. If only two coefficients are considered, the ratio $\mathrm{SBR}=\sigma$. However, in general,

$$
r_{\min } \sigma \leqslant \mathrm{SBR} \leqslant \sigma r_{\max }
$$

where $r_{\min }$ and $r_{\max }$ are the minimum and maximum values of ratio of quadratic forms respectively.

The area of the phase-space resolution cell is given by SBP. And the SBR dictates the ratio of the sides of the cell. Many possibilities arise (which are analysed completely [26]). For instance, when $\sigma$ is large (and hence the spectral window is in the low frequency part of the spectrum), and the coefficients $\gamma_{n} s$, are such that the term inside the square root symbol of eqn. 11 is small, the spectral width is also small. On the contrary, when $\sigma$ is small (and hence the spectral window is in the high frequency part of the spectrum), the $\eta_{s} s$ may assume values such that the term inside the square root symbol of eqn. 11 is large. As a consequence, the spatial width could be large in the high frequency part of the spectrum.

In the classical wavelet framework, the shape of the resolution cell, in phase-space depends on the scale. The resolution in the spatial domain increases (decreases in the frequency domain) with an increase in the scale parameter. The area within each resolution cell is the same. In the new vector wavelet framework, on the contrary, the shape of the resolution cell does indeed depend on the value of $\sigma_{i}, \mathrm{i}=0,1, \ldots, N$, but the area within the resolution cell varies depending on the nature of the signal. See Fig. 3. It is possible to choose a small value of $\sigma$ and control the coefficients $\gamma_{i}$ s such that the phasespace resolution cell has a spatial length which is larger than its spectral width. On the contrary, by choosing larger value of sigma, it is still possible to realise a phasespace resolution cell whose spatial length is smaller than its spectral width. The inference is that the shape of the resolution cell is variable, independent of its location in the phase-space.
Property 3: Zero crossings at various scales: Using the recursive relations for Hermite polynomials, the second

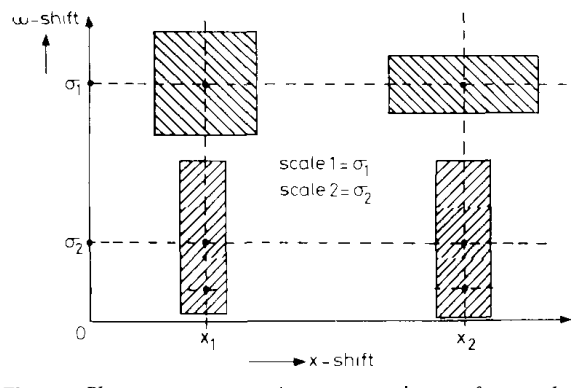

Fig. 3 Phasr-space representation in new wavelet arrayframework

derivative ('Laplacian') of the signal representation at any scale $(\sigma)$ can be shown to be given by

$$
\begin{aligned}
& \left(\frac{\partial^{2}}{\partial x^{2}}\right)_{\sigma} f(x) \\
& \quad \simeq \sum_{n} i_{n}\left[\frac{1}{4 \sigma} H_{n+2}-\left(\frac{1}{2 \sigma}+\frac{n}{\sigma}\right) H_{n}+\frac{n(n-1)}{\sigma} H_{n-2}\right] \\
& \quad=\sum \mu_{n} H_{n}
\end{aligned}
$$

where the subscript $\sigma$ under the partial derivative denotes that the derivative is taken at scale $\sigma$, and

$$
\begin{gathered}
\mu_{n}=\sum_{n} \frac{1}{4 \sigma} \gamma_{n-2}-\left(\frac{1}{2 \sigma}+\frac{n}{\sigma}\right) \gamma_{n} \\
\frac{(n+2)(n+1)}{\sigma} \gamma_{n+2}
\end{gathered}
$$

The 'Laplacian' of the various layers of the approximated signal can be obtained merely by substituting the wavelet coefficients. This provides an elegant way of extracting the zero crossings at different scales of the representation.

Property 4: Layered decomposition: The decomposition in terms of layers at different scales has the desired property of capturing independent spectral information, by adjusting the scale parameter $\sigma$. By virtue of the multistage decomposition, the spectrum of the first level approximation is distinct from that of the second level, and the spectrum of the second approximation is distinct from that of the third level, and so on. It can be shown that the signal outputs of the various layers are independent.

Remark 3.5: As far as the application of the proposed representation is concerned, the following points (which are based on the computer implementation)* are believed to be helpful.

The start value for $\sigma$ can be obtained from the spacebandwidth-ratio of the given signal (in the case that the signal is concentrated around $x=0$ in the spatial/time 
which account for the large errors in reconstruction at the boundaries.

The corresponding results for $\mathbf{2 D}$ are shown in Figs. thetic for a $\begin{array}{r}\text { and Figs. 6A for a syn- } \\ \text { In the latter illustration, }, \text { that the }\end{array}$ gr value of the gray levels in the ll image is not the same as that in the reconstruction:' and that the $i \quad f$ changes at $\in \quad l$ are not reconstructed satisfactorily

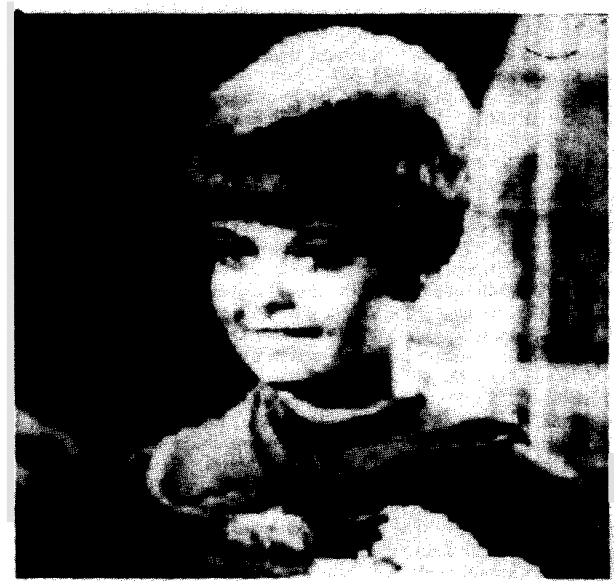

Fig. 5A Original image (naturah

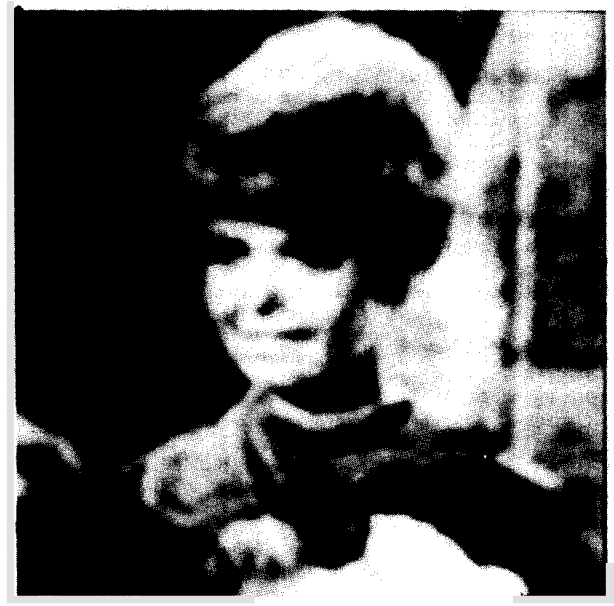

Fig. 5B Reconstructed image using seven layers

It has been found, in the course of orthogonalising the outputs of the layers, for both 1D and 2D signals, that the redundancy in the outputs of the layers is not considerable. This shows that the new scheme possesses, in addition to the multilayered structure, data compression properties also.

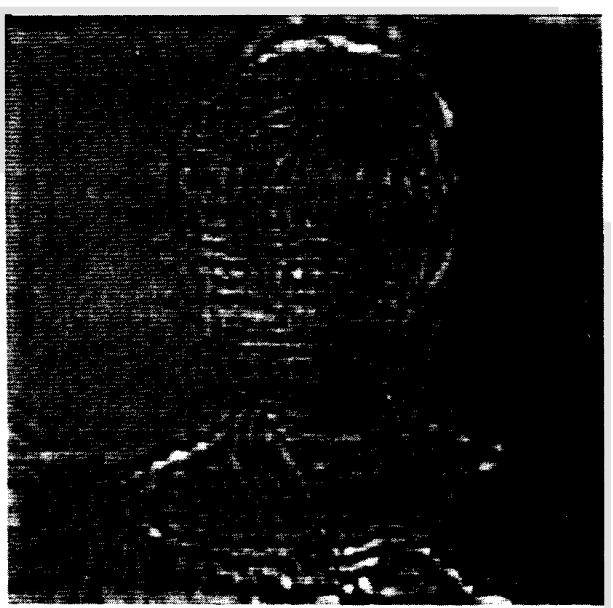

Fig. 5C Residual image (error)after seven layers

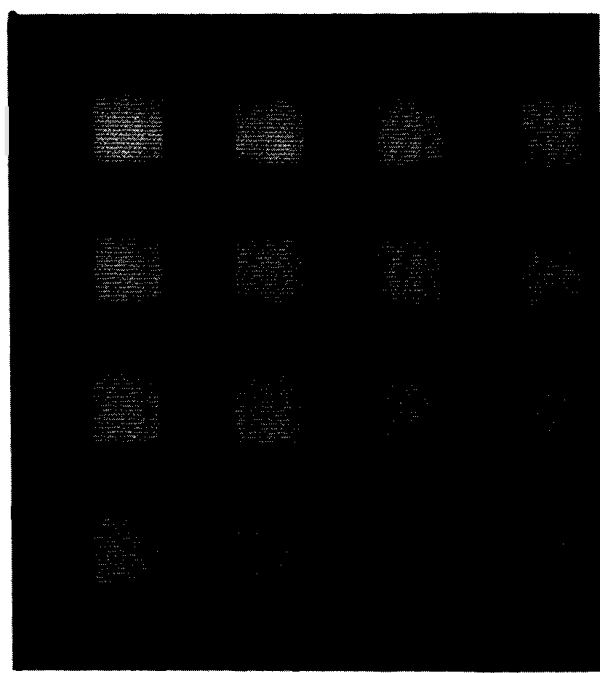

Fig. 6A Original image (synthetic)

\section{Conclusions}

Representation schemes meant for localisation of information in $1 \mathbf{D}$ and $2 \mathbf{D}$ signals have been reviewed. A new and elegant method of signal representation based on a wavelet-like array has been proposed. This involves the use of 1 and 2D generalised Hermite polynomials, which are orthogonal, for 1 and 2D signals, respectively. The novelty of the results lies in the fact that the traditional assumption of compact support in the spatial or frequency domain has been dispensed with. An important 
byproduct is that an upper bound on the spacebandwidth product (or 'uncertainty') is specified. A chal. lenging problem is to derive an optimum sampling scheme for the choice of the scale parameter for both one and two dimensional signals.

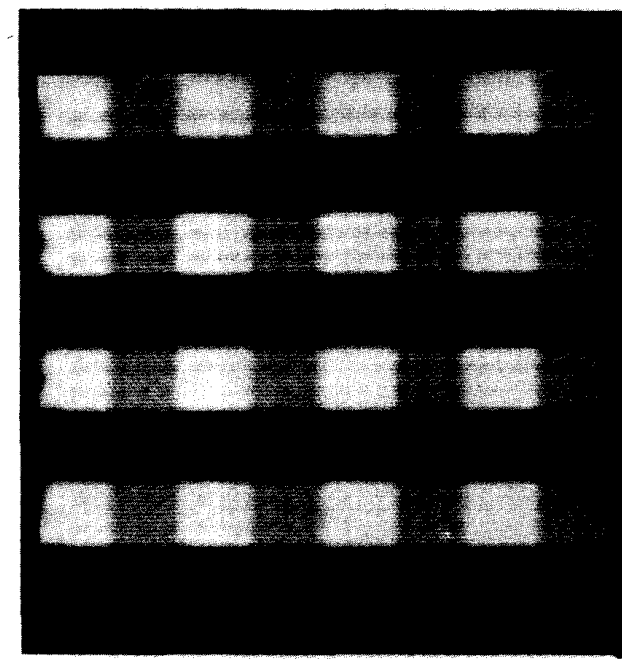

Fig. 6B Reconstructed image using seven layers

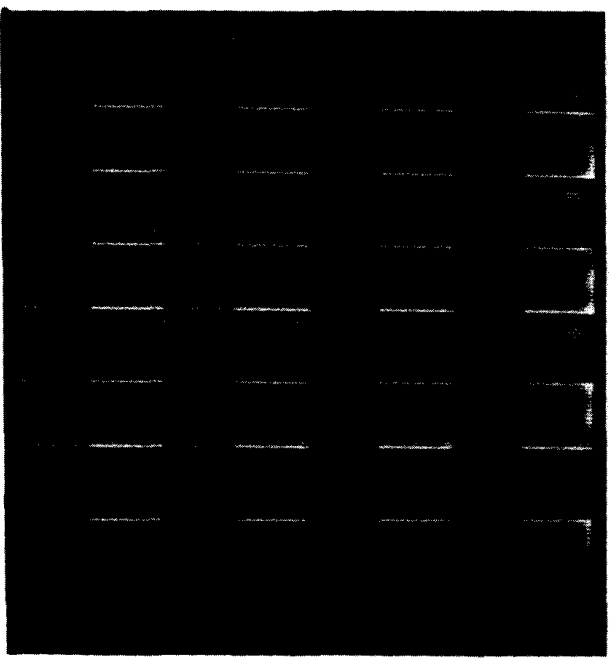

Fig. 6C Residual image (error) after seven layers

\section{$7 \quad$ References}

I DAUGMAN, J.: 'Nonorthogonal wavelet representations in relaxation networks: image encoding and analysis with biological visual pnmitives', in TAYLOR, J., and MANNION, C. (Eds.): 'New developments in neural computing' (Institute of Physics Press, 1989), pp.

2 DAUGMAN J.: 'Two-dimensional spectral analysis of cortical

3 MARR, D., ULLMAN, S., and POGGIO, T.: 'Bandpass channels, ero-crossings, and early visual information processing', J. Opt.Soc. Am., 1979, 69, (6), pp. 914-916

HUBEL, D.H., and WIESEL, T.N.: 'Receptive fields, binocular interaction and functional architecture in the cat's visual cortex', J. Fhysiul., 1962,160, pp. 106-154

5 WTRIN, A. 'Scale space filtering'. Proceedings of the International joint cis, A :

CURTIS, S.R., OPPENHEIM, A.V., and LIM, J.S.: 'Signal reconstruction from Fourier transform sign information', IEEE Trans. 1985, ASP-33, (3), pp. 613-657

7 MARR, D.: 'Vision' (Freeman, San Francisco, CA, 1982)

8 LEVINE, M.D.: 'Vision in man and machine' (McGraw-Hill, New

York, 1985), pp. 59-99

BURT, P.J.. and ADELSON, E.H.: 'The Laplacian pyramid as a compact image code , ILEE Trans., 1983, COM-31, pp. 532-540

UNSER, M.: 'An improved least squares Laplacian
image compression', Signal Proc., 1992, 21, pp. 187-203

I1 GABOR, D.: 'Theory of communication', J. Inst. Elec. Eng. (Lond.), 1946, 93, (III), pp. 429-457

12 BASTIAANS, M.J.: 'Gabor's signal expansion and degrees of freedom of a signal ${ }^{,}$, Proc. IEEE, 1980, 68, pp. 538-539

13 GROSSMAN, A., and MORLET, J.: 'Decomposition of Hardy functions into square integrable wavelets of constant shape', SIAM J. Math., 1984, 15, pp. 723-736

14 MALLAT, S.: 'Multi-resolution approximation and wavelet orthonormal bases of $L^{2}$, Trans. Am. Math. Soc., 1989a, 3-15, pp. 69-87, 'A theory for multiresolution signal decomposition: the wavelet rep-; resentation', IEEE Trans., 1989b, PAMI-11, (7), pp. 674-693; 'Multifrequency channel decompositions of images and wavele models', IEEE Trans., 1989, ASP -37, (12), pp. 2091-2110

15 DAUBECHIES, I.: 'The wavelet transform, timefrequency localisation and signal analysis', IEEE Trans., 1990, IT-36, (5), pp. 9611004: 'Orthonormal basis of compactly supported wavelets', Commun. Pure Appl. Math., 1988, 41, pp. 909-996

$16 \mathrm{ZAK}$, J.. 'The kq-representation in the dynamics of electrons in solids, solid state physics', in 'Advances in research and applications' (Academic Press, 1972), 27, pp. 1-62

17 DAUBECHIES. I., GROSSMANN, A., and MEYER, Y.: 'Painless nonorthogonal expansions', J. Math. Phys., 1986, 21, (5), pp. $1271-$ 1283

18 KUMAR. A., FUHRMANN, D.R., FRAZIER, M., and JAWERTH, B.D.: 'A new transform for timefrequency analysis', IEEE Trans., 1992,SP-40, (7), pp. 1697-1707

19 NEWLAND. D.E.: 'Harmonic wavelet analysis', Proc. Roy. Soc. Lond. A, 1993,443, pp. 203-225

20 DONOHO, D.L., and STARK, P.B.: "Uncertainty principles and signal recoverv'. SIAM J. Aool. Math., 1989. 49, (3). Dp. 906-931

21 DE BRUIJN, N.G.: 'Uncertainty principles in Fourier analysis', SHISHA, O.(Ed.): 'Inequalities' (Academic Press, New York, 1967), pp. $57-71$

22 PAPOULIS, A.: 'Signal analysis' (McGraw-Hill, 1986)

23 LEBEDEV, N.N.: 'Special functions and their applications' (Dover

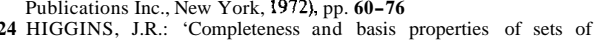
special functions' (Cambridge University Press, Cambridge, Great

25 Britain, 1977) 'Orthogonal polynomials' (American Mathematical Society, 1975

26 VENKATESH, Y.V., RAMANI, K., and NANDINI, R.: (a) 'Vector wavelet decomposition of images using a Hermite sieve',
SADHANA, Special issue Comp. Vision, J. Ind. Acad. Sci., 1992, in SADHANA, Special issue Comp. Vision, J. Ind. Acad. Sci., 1992, in
press $(\boldsymbol{b})$; 'Image representation using vector wavelets'. 1 Ith IAPR press $(b)$; 'Image representation using vector wavelets'. 1 Ith IAPR
international conference on pattern recognition and image prointernational conference on pattern recognition and image pro-
cessing, The Hague, The Netherlands, Aug.-Sept. 1992;(c) 'Wavelet cessing, The Hague, The Netherlands, Aug,-Sept. 1992; (c) 'Wavelet
array decomposition for signal representation using generalised Hermite polynomials'. MSo(Eng.) thesis, Department of Elec. Eng. Hermite polynomials'. MSo(Eng.) thesis, Department of Elec. Eng.
IISc. 1992 IISc, 1992

27 CALWAY, A.: 'The multiresolution Fourier transform'. PhD thesis,
University of Warwick, 1989 